Proc. of the 15th Int. Workshop on Slow Positron Beam Techniques and Applications, Prague, September 2-6, 2019

\title{
Limitations on the Positron Lifetime Spectra Decomposability Applying the Iterative Least-Square Re-Convolution Method Using the Instrumental Responses Obtained from ${ }^{207} \mathrm{Bi}$ and ${ }^{60} \mathrm{Co}$
}

\author{
D. PetschKe ${ }^{a, *}$, R. $\operatorname{Helm}^{b}$, M. HAAKS ${ }^{c}$ And T.E.M. StaAB ${ }^{a}$ \\ ${ }^{a}$ Department of Chemistry and Pharmacy, University Würzburg, 97070 Würzburg, Germany \\ ${ }^{b}$ Department of Aerospace Engineering, Universität der Bundeswehr München, 85577 Neubiberg, Germany \\ ${ }^{c}$ AERO-LASER GmbH, Gesellschaft für Gasanalytik, 82467 Garmisch-Partenkirchen, Germany
}

\begin{abstract}
Since the decomposition of positron lifetime spectra requires solving an ill-posed and inverse problem, the accurate knowledge of the spectrometer's instrument response function is crucial for extracting the true underlying physical information of the phenomenon under investigation. In general, the instrument response function is modelled by a superposition of Gaussian functions, since an analytical solution for the convolution with an exponential distribution function exists and, hence, the characteristic lifetimes and its corresponding contributions can be obtained by non-linear least-squares fitting. In contrast, the iterative least-squares re-convolution approach determines the best fit of the recorded lifetime spectrum by re-convoluting a sum of $N$ expected exponential decays with the numerical data of the experimentally obtained instrument response function. For a laboratory setup, two methods exist to estimate the shape of the instrument response function from experiment: (1) the direct method using a ${ }^{60} \mathrm{Co}$ isotope and an indirect method by graphically deconvoluting the monoexponential lifetime spectrum obtained from ${ }^{207} \mathrm{Bi}$. For both variants, the energies of the incident gamma-rays are considerably different to the energies accompanying the creation $(1274 \mathrm{keV})$ and annihilation $(511 \mathrm{keV})$ of a positron using ${ }^{22} \mathrm{Na:}{ }^{60} \mathrm{Co}(1173 \mathrm{keV}$, $1333 \mathrm{keV}),{ }^{207} \mathrm{Bi}(570 \mathrm{keV}, 1064 \mathrm{keV})$. Here we present a detailed study on the basis of plastic scintillators regarding the spectra decomposability by using the re-convolution technique with experimentally obtained instrument response functions. We can clearly show that beyond incident gamma-ray energy differences, the Compton scattering effects and pile-up events represent the limiting factors in this approach.
\end{abstract}

DOI: 10.12693/APhysPolA.137.171

PACS/topics: positron lifetime spectroscopy, data analysis, ill-posed problem, multi-exponential decay, inverse problem

\section{Introduction}

Positron annihilation lifetime spectroscopy (PALS) provides a power tool for non-destructive microstructure investigations on the atomic scale in a broad field of material classes ranging from metals and light alloys to semiconductors, polymers, and glasses.

The individual lifetimes of the positrons follow an exponential distribution, which can be expressed as

$$
f(t)=A \exp \left(-\frac{t}{\tau}\right)
$$

with the decay amplitude $A$ given by the number of counts and the time constant of the decay defining the characteristic lifetime $\tau$ as a function of the electron density localized at the annihilation site [1] associated to the kind and size of open-volume defects such as vacancies or pores. However, the extraction of the relevant physical information from multi-exponential decays of which constitute the solution $\left(A_{i}\right.$ and $\left.\tau_{i}^{-1}\right)$ may appear uncom-

\footnotetext{
* corresponding author; e-mail: danny petschke@uni-wuerzburg.de
}

plicated at the first glance but describes one of the oldest and intensively studied ill-posed problems [2], since the uniqueness or even the existence of its solution is not always assured and further the solution may not depend continuously on the data [3].

Moreover, the experimentally obtained (or real) lifetime spectrum

$$
F_{\exp }(t)=\left(g * \sum_{i=0}^{N-1} f_{i}\right)(t)
$$

appears convoluted with the instrument response function (IRF) $g$ known to be (possibly) not directly (or even indirectly) accessible from the measurement. Therefore, this ill-conditioned problem becomes additionally inverse.

Due to these essential difficulties, an accurate estimation of the IRF is crucial for a profound spectra analysis, which is commonly realized by a superposition of Gaussian functions, since an analytical solution for Eq. (2) exists $[4,5]$. It is obvious that errors are transmitted to the characteristic lifetimes $\left(\tau_{i}\right)$ and its corresponding contributions $\left(I_{i}=A_{i} / \sum_{i=0}^{N-1} A_{i}\right)$ if the model of the underlying IRF is not determined correctly.

However, in the present study we discuss the limitations of the decomposability on a discrete lifetime spectrum obtained from high-purity (5N) $\alpha$-Fe by using (1) 
the directly measured prompt spectrum from the ${ }^{60} \mathrm{Co}$ isotope and (2) the indirectly determined IRF from a monoexponential decay spectrum obtained from ${ }^{207} \mathrm{Bi}$ with a "known" natural decay time of $\tau=182 \mathrm{ps}$. Since the commonly used non-linear least-squares fitting (NLSF) technique requires the analytical description of the model function (Eq. (2)) [4, 5], we apply the iterative leastsquare re-convolution method, which determines the best fit by re-convoluting a sum of $N$ expected components (Eq. (1)) with the (real) experimentally obtained data from ${ }^{60} \mathrm{Co}$ or ${ }^{207} \mathrm{Bi}$ using the convolution theorem. This theorem states that the Fourier transform of a convolution of two functions (Eq. (2)) is the pointwise product of their Fourier transforms. Therefore, Eq. (2) can be equivalently expressed as

$$
\begin{gathered}
F_{\exp }(t)=\mathcal{F}^{-1}\left\{\mathcal{F}\{g\} \cdot \mathcal{F}\left\{f_{0}\right\}\right\}+\cdots \\
+\mathcal{F}^{-1}\left\{\mathcal{F}\{g\} \cdot \mathcal{F}\left\{f_{N-1}\right\}\right\}
\end{gathered}
$$

This technique is commonly used for the analysis of fluorescence lifetime spectra (FLS) (see e.g. [6]).

\section{Experimental setup, and lifetime spectra acquisition and analysis software}

The spectra presented here were acquired in a colinearly arranged $\left(180^{\circ}\right)$ two-detector (2D) configuration setup as described elsewhere by the authors [7]. DDRS4PALS v1.08 [8, 9] served as a software tool for the acquisition and simulation of the lifetime spectra, where the integrated simulation functionality is provided by DLTPulseGenerator library (v1.3) [10-13]. Furthermore, the raw detector anode-pulses were filtered prior to the generation of the spectra according to its area and shape allowing rejection of pile-up events and multiple occurrences of pulses within the corresponding digitizer readout range (so-called sweep) [9, 14-17]. For the lifetime spectra analysis using the re-convolution approach, the authors provide the python-based software DLTReconvolution v.1.2 [18], which allows in addition the extraction of the IRF from a monoexponential decay spectrum $f$ obtained from ${ }^{207} \mathrm{Bi}$ using the graphical deconvolution method as shown by Köchlin et al. [19]:

$$
\mathrm{IRF}_{i}=f_{i}+\frac{\tau}{h} \frac{f_{i+1}-f_{i-1}}{2},
$$

where $\tau$ is the single characteristic lifetime, and $h$ is the channel width.

The isotopes which served in this study had the following activities: ${ }^{22} \mathrm{Na}$ sealed in kapton foil $(25 \mu \mathrm{Ci}),{ }^{207} \mathrm{Bi}$ $(1 \mu \mathrm{Ci})$, and ${ }^{60} \mathrm{Co}(1 \mu \mathrm{Ci})$.

\section{Sensitivity check of the re-convolution technique on a simulated positron lifetime spectrum}

To mimic the experimental lifetime spectrum obtained from high-purity $\alpha$-iron (first component, $\tau_{1}=107.5 \mathrm{ps}$ ) as shown in the forthcoming Sect. 4, a 3-component lifetime spectrum (4 Mcounts) with a single Gaussian IRF $(\mathrm{FWHM}=235.0 \mathrm{ps})$ has been simulated.

\section{TABLE I}

Left: parameters serving as input for the positron spectra simulation (input). Right: parameters obtained from the re-convolution fit (output).

\begin{tabular}{c|c|c|c|c}
\hline \hline \multirow{2}{*}{ Component $i$} & \multicolumn{2}{|c|}{ Input } & \multicolumn{2}{c}{$\begin{array}{c}\text { Output: } \\
\text { re-convolution fit }\end{array}$} \\
\cline { 2 - 5 } & $\tau_{i}[\mathrm{ps}]$ & $I_{i}[\%]$ & $\tau_{i}[\mathrm{ps}]$ & $I_{i}[\%]$ \\
\hline 1 & 107.5 & 82.5 & $107.4(0.3)$ & $82.5(0.3)$ \\
2 & 355 & 17.1 & $353.8(2.2)$ & $17.1(0.2)$ \\
3 & 2500 & 0.4 & $2409(119)$ & $0.418(0.011)$ \\
\hline IRF model & \multicolumn{2}{|c|}{ Gaussian } & \multicolumn{2}{|c}{-} \\
FWHM [ps] & \multicolumn{2}{|c|}{235.0} & \multicolumn{2}{|c|}{-} \\
$\chi_{v}{ }^{2}$ & \multicolumn{2}{|c|}{-} & \multicolumn{2}{|c|}{0.978}
\end{tabular}

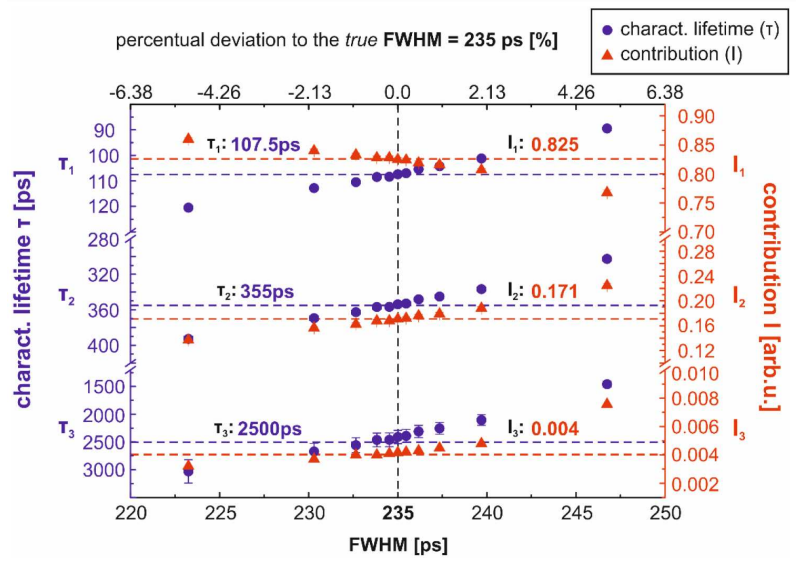

Fig. 1. Mapping of the retrieved parameters $(\tau, I)$ from the simulated 3-component lifetime spectrum (Table I) using the re-convolution fit with IRFs of varying FWHMs with respect to the true underlying FWHM (=235.0 ps).

The second component $\left(\tau_{2}=355 \mathrm{ps}\right)$ is related to the combined characteristic lifetime originating from annihilation events in the sodium $\left({ }^{22} \mathrm{Na}\right)$ and its covering kapton foil, whereas the long and low-contributed characteristic lifetime $\tau_{3}(=2500 \mathrm{ps})$ indicates the complex source component originating either from the glue or the positronium which is probably forming at interfaces between the source and the covering kapton foil. The input parameters are given in Table I (column "Input").

As indicated by Table I, fit parameter values (column "Output: re-convolution fit") in good agreement $\left(\chi_{v}{ }^{2}=0.978\right)$ with the true underlying information (column "Input") can be obtained by applying the reconvolution technique on spectra accomplished under perfect conditions, meaning that setup and method allow the direct and rather accurate measurement (or determination) of the true IRF (FWHM = 235.0 ps).

To determine the sensitivity of the re-convolution fit regarding the accurate decomposability in terms of deviations to the true underlying IRF, 10 simulated prompt spectra having deviations of $\pm 0.2(0.5,1.0,2.0,5.0) \%$ with respect to the true FWHM (= $235.0 \mathrm{ps)}$ were applied for the spectra analysis. As clearly seen in Fig. 1, 
errors are already transmitted to the retrieved information $(\tau, I)$ at only slight deviations of about $\pm 0.5 \%$ in the FWHM $(=233.8 / 236.2 \mathrm{ps})$. Moreover, a clear tendency in the characteristic lifetimes $(\tau)$ can be observed: positive $(+)$ deviations in the FWHM compensate the decay slopes (log-scale slope $\lambda \sim \tau^{-1}$ ), meaning that the characteristic lifetimes tend to significant smaller values, whereas negative deviations $(-)$ perform contrarily. For the corresponding contributions $I_{1}$ and $I_{2}$, the opposite behavior can be observed.

\section{Decomposition of an experimentally obtained lifetime spectrum on high-purity $\alpha$-Fe}

In a first step, ${ }^{60} \mathrm{Co}$ has been used to determine the gamma-ray energy-dependence of the setup resolution, i.e., the resulting FWHM of the setup IRF, by incrementally shifting a constant window of size $48.8 \mathrm{mV}$ with a step width of $24.4 \mathrm{mV}$ over the relevant PHS region ([48.8;268.4] $\mathrm{mV})$ covering the START $([63.4 ; 107.4] \mathrm{mV})$ and STOP ([224.5;268.4] mV) windows subsequently applied for the experiment (see Fig. 2, grey shaded region). In the course of this, it is assumed that the pulse height spectrum (PHS) as shown in Fig. 2a and c is equivalent for both PMTs and attached plastic scintillators (symmetric setup condition) and further indicates high linearity between the incident (or rather Compton scattered) photon energy and pulse amplitude $A[\mathrm{mV}]$ in the relevant PHS region (see Fig. 2, grey shaded region). Further assuming that this dependence is mainly dominated by the transit time spread (TTS) of the PMTs, which is known to be indirectly proportional to the square root of the number of photoelectrons per pulse [20], and that this conversion is exclusively accomplished by single Compton scattering, the following linear representation (Fig. 2b, solid line):

$$
\frac{1}{\text { FWHM }^{2}}\left[\mathrm{ps}^{-2}\right]=1.17 \times 10^{-7} A[\mathrm{mV}]+2.26 \times 10^{-6}
$$

was determined and subsequently used to estimate the setup resolutions for the different isotopes as given

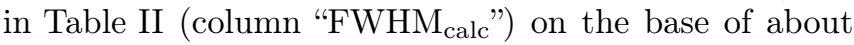
50000 correlated (START/STOP) events modelling the

TABLE II

Left: spectra statistics acquired. Middle: calculated setup resolutions according to Eq. (5) $\left(\mathrm{FWHM}_{\text {calc }}\right)$ representing the energy distributions in the PHS windows as shown in Fig. 2c. Right: setup resolutions resulting from the fit $\left(\mathrm{FWHM}_{\mathrm{fit}}\right)$ on the experimentally obtained IRFs using a single Gaussian function.

\begin{tabular}{c|c|c|c}
\hline \hline & $\begin{array}{c}\text { Spectra } \\
\text { statistic } \\
{[\text { Mcounts }]}\end{array}$ & $\begin{array}{c}\text { FWHM }_{\text {calc }}[\mathrm{ps}] \\
(\text { Eq. (5)) }\end{array}$ & $\begin{array}{c}\mathrm{FWHM}_{\text {fit }}[\mathrm{ps}] \\
(\text { single } \\
\text { Gaussian fit) }\end{array}$ \\
\hline${ }^{22} \mathrm{Na}$ & $\approx 4$ & $240.2(0.4)$ & $245.1(0.6)$ \\
${ }^{60} \mathrm{Co}$ & $\approx 2.5$ & $239.7(0.4)$ & $239.6(0.3)$ \\
${ }^{207} \mathrm{Bi}$ & $\approx 5$ & $240.5(0.3)$ & $227.9(0.5)$
\end{tabular}
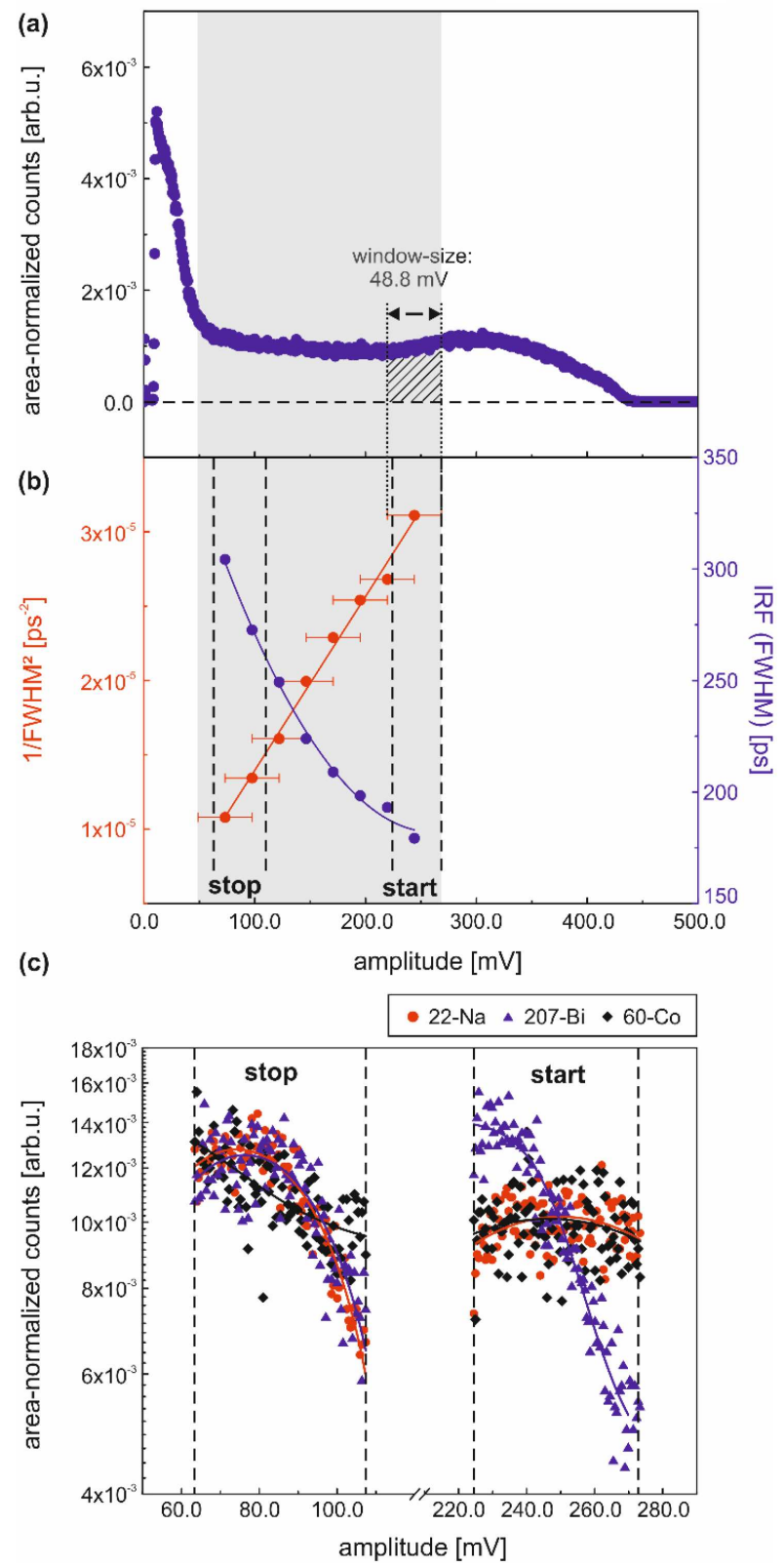

Fig. 2. (a) Pulse height spectrum (PHS) of plastic scintillators (BC422Q0.5) for ${ }^{60} \mathrm{Co}$. (b) Energydependence of the setup resolution (FWHM) obtained from 8 regions of the ${ }^{60} \mathrm{Co}$ PHS (a) each indicated by the $x$-error bars. (c) PHS for all three isotopes $\left({ }^{22} \mathrm{Na}\right.$, ${ }^{207} \mathrm{Bi}$ and ${ }^{60} \mathrm{Co}$ ) each consisting of about 50000 correlated START/STOP events. The solid line indicates the respective moving average serving as guide for the eye.

energy distributions (PHS) as displayed in Fig. 2c. As is clearly seen, the calculated FWHMs of the ${ }^{60} \mathrm{Co}$ $(=239.7 \pm 0.4 \mathrm{ps})$ and ${ }^{207} \mathrm{Bi}(=240.5 \pm 0.3 \mathrm{ps})$ isotopes fully reflect the resolution obtained from the ${ }^{22} \mathrm{Na}$ isotope $(=240.2 \pm 0.4 \mathrm{ps})$ within their uncertainties and, thus, expecting in theory a reliable spectra decomposition by this approach under the above given assumptions. 

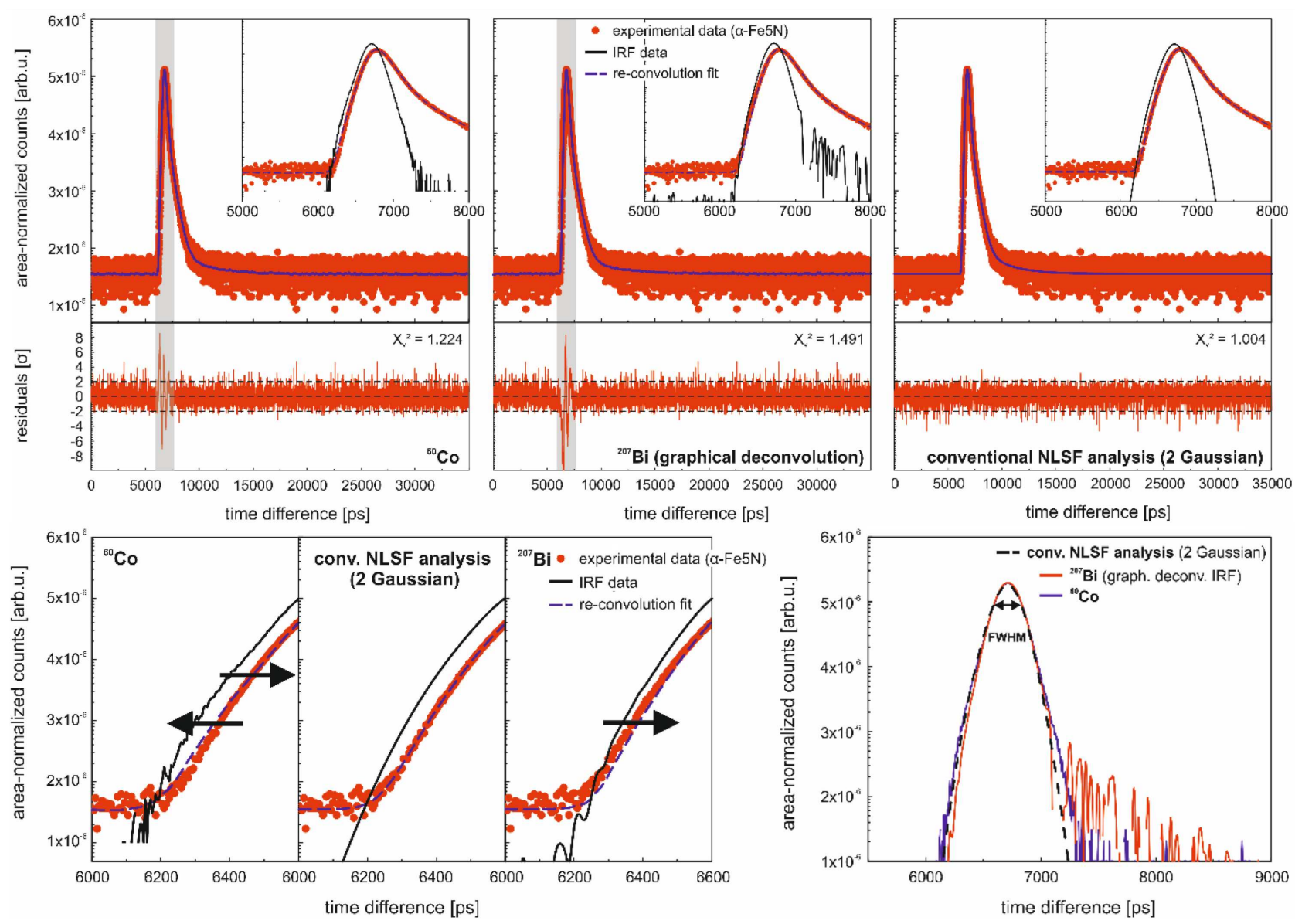

Fig. 3. (a)-(c) Experimentally obtained lifetime spectrum on $\alpha$-Fe5N (dots) and corresponding IRF (solid line), which served for the 3-component analysis (Table III) using the re-convolution technique. The dashed lines indicate the respective model fits, whereas the plots at the bottom show the corresponding residuals. (d) Onset regions of plots (a)-(c). (e) Collection of all IRFs from (a)-(c) for comparison.

Since the graphical deconvolution technique according to Eq. (4) is evidently highly sensitive to noise, the Savitzky-Golay filtering (2nd order polynomial, window size $=11$ channels) has been applied in a previous stage for data smoothing, where the smoothing parameters were prior determined and verified on simulated spectra under equivalent conditions in terms of statistics $(\approx 5$ millions) and binning $(h=5 \mathrm{ps})$. A discussion regarding the graphical deconvolution technique applied on the monodecay spectrum of ${ }^{207} \mathrm{Bi}$ will be part of the forthcoming publication by the authors.

Furthermore, to provide comparability in the retrieved parameters and goodness-of-fit $\left(\chi_{v}{ }^{2}\right)$ according to the commonly applied NSLF technique, the resulting IRF modelled by 2 Gaussian functions was subsequently used for the re-convolution fit as shown in Fig. 3c indicating a good agreement between experiment and model $\left(\chi_{v}^{2}=1.004\right)$.

As it can be observed in Table II (column "FWHM fit ), the FWHM $(=245.1 \pm 0.6 \mathrm{ps})$ of the IRF obtained from ${ }^{22} \mathrm{Na}$ (2 Gaussian model) indicates an additional broadening with respect to the FWHM
(=240.2 $\pm 0.4 \mathrm{ps})$ semitheoretically determined accord-

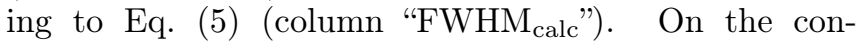
trary, the FWHM $(=227.9 \pm 0.5 \mathrm{ps})$ of the IRF indirectly obtained from ${ }^{207} \mathrm{Bi}$ tends to significant lower values, whereas for ${ }^{60} \mathrm{Co}$ the FWHM $(=239.6 \pm 0.3 \mathrm{ps})$ behaves as expected (Eq. (5)). As indicated in Table III, these deviations in the IRF broadening of ${ }^{60} \mathrm{Co}$ $(=239.6 \pm 0.3 \mathrm{ps})$ and ${ }^{207} \mathrm{Bi}(=227.9 \pm 0.5 \mathrm{ps})$ with regard to the FWHM $(=245.1 \pm 0.6 \mathrm{ps})$ of ${ }^{22} \mathrm{Na}$ fully reflect the tendencies in the retrieved parameter values $(\tau, I)$ as produced by the simulations in Fig. 1, thereby assuming that the 2 Gaussian IRF model approximates the true underlying IRF best (Fig. 3c).

However, these broadening differences between the isotopes cannot be exclusively described by different incident (or Compton scattered) photon energies at this point and might be due to the following effects:

Even though the energy distributions are equal for the three isotopes (Fig. 2c and Table II, 1st column), the resulting photon (and electron) propagation angles after the Compton scattering are differently distributed for the applied PHS windows, since the Compton edges 
TABLE III

Retrieved parameters $(\tau, I)$ from the re-convolution fit and corresponding goodness-of-fit $\left(\chi_{v}^{2}\right)$ for the different IRFs. The mean values $\left(\Delta t_{1,2}\right)$ of the 2 Gaussian functions representing the IRF obtained from the conventional NLSF technique are given with respect to $t_{0}=6700 \mathrm{ps}$

\begin{tabular}{l|c|c|c}
\hline \hline & $\begin{array}{c}\text { NLSF technique } \\
(2 \text { Gaussian } \\
\text { model) }\end{array}$ & IRF: ${ }^{60} \mathrm{Co}$ & $\begin{array}{c}\text { IRF: }{ }^{207} \mathrm{Bi} \\
\text { (graph. } \\
\text { deconvolution) }\end{array}$ \\
\hline$\tau_{1}: \alpha$-Fe [ps] & $106.4(1.2)$ & $108.6(0.4)$ & $120.0(0.4)$ \\
$I_{1}: \alpha$-Fe [\%] & $82.7(0.4)$ & $84.4(0.4)$ & $87.1(0.4)$ \\
$\tau_{2}:$ source [ps] & $358.5(3.3)$ & $374.8(3.1)$ & $403.4(4.1)$ \\
$I_{2}:$ source [\%] & $16.9(0.2)$ & $15.3(0.3)$ & $12.6(0.3)$ \\
$\tau_{3}:$ complex & & & \\
$\quad$ source [ps] & $2425(168)$ & $2642(210)$ & $2875(307)$ \\
$I_{3}:$ complex & & & \\
$\quad$ source [\%] & $0.391(0.002)$ & $0.314(0.005)$ & $0.263(0.001)$ \\
\hline IRF $_{1}:$ & $45.3(2.3)$ & - & - \\
$I_{1}[\%]$ & $305.31(1.62)$ & & \\
$\mathrm{FWHM}_{1}[\mathrm{ps}]$ & $-6.41(3.09)$ & & \\
$\Delta t_{1}[\mathrm{ps}]$ & & & \\
\hline $\mathrm{IRF}_{2}:$ & $54.7(2.3)$ & - & \\
$I_{2}[\%]$ & $212.56(2.11)$ & & \\
$\mathrm{FWHM}_{2}[\mathrm{ps}]$ & $17.65(0.56)$ & & \\
$\Delta t_{2}[\mathrm{ps}]$ & 1.004 & 1.224 & 1.491 \\
\hline$\chi_{v}{ }^{2}$ & & &
\end{tabular}

appear at unequal energies $\left({ }^{60} \mathrm{Co}: 963.4 / 1118.1 \mathrm{keV}\right.$; ${ }^{207} \mathrm{Bi}$ : $393.3 / 857.7 \mathrm{keV}$; ${ }^{22} \mathrm{Na}$ : $\left.340.7 / 1061.2 \mathrm{keV}\right)$, meaning that different scatter angles and different scatter positions in the scintillator cause deviations in the average travel distance and delay between photon incident and detection.

Therefore, the better timing resolution of ${ }^{207} \mathrm{Bi}$ with respect to ${ }^{60} \mathrm{Co}$ might be explained by the fact that low scattering angles, i.e., photon energies near the Compton edges, are producing an effectively lower jitter in the average travel time and distance. Moreover, the differential cross-sections of the Compton scattered photons behave differently for the gamma-ray energies of the three isotopes which might also contribute to the timing jitter.

Nevertheless, these effects do not explain the deviation in the IRF broadening between ${ }^{60} \mathrm{Co}$ and ${ }^{22} \mathrm{Na}$, since one rather would expect a FWHM for ${ }^{22} \mathrm{Na}$ tending to lower values as for ${ }^{207} \mathrm{Bi}$ due to their close Compton edge energies in the STOP window region $\left({ }^{207} \mathrm{Bi}: 393.3 \mathrm{keV}\right.$; $\left.{ }^{22} \mathrm{Na}: 340.7 \mathrm{keV}\right)$. These deviations might be due to erroneous detection of single annihilation events (e.g., PMT 1: start - PMT 2: start + stop) or summation of signals causing pile-up events, which cannot be fully suppressed by software-based filtering, considering that ${ }^{22} \mathrm{Na}$ was available in a significantly $(25 \times)$ higher activity $(25 \mu \mathrm{Ci}$ vs. $1 \mu \mathrm{Ci}$ ) and additionally generated at least three photons per contributing event evidently producing a higher probability of signal-misinterpretation. Further studies will include different detector arrangements (and source positions) and the usage of comparable activities for the isotopes $(1-5 \mu \mathrm{Ci})$ to verify this hypothesis in the given context.

Finally, deviations between the isotopes are not only present for the broadening of the IRF but also for their shape, as can be observed in Fig. 3c (and e), since differences between model fit and data in the onset spectra region do not behave according to the IRF broadening (Table II). This effect might result from an insufficient reproducibility of the exact placement for the different isotopes in between the detector arrangement causing variations in the relative effective area of illumination of the PMT photocathode, which finally influences the transit time, i.e., the time-zero point of the obtained spectrum, and additionally the broadening of the TTS, i.e., the FWHM of the resulting IRF [20]. Further studies will investigate the sensitivity on the isotope relative displacement with respect to a reference position regarding the resulting shape and FWHM of the IRF.

\section{Conclusion and outlook}

\subsection{Simulation}

In this study, we have first verified the re-convolution technique on simulated PALS spectra implementing perfect conditions, i.e., method and setup allow the direct measurement of the underlying true IRF. Furthermore, it was shown that the spectra decomposition in terms of extracting the true underlying physical information is highly sensitive to the exact knowledge of the IRF, since already slight differences in the broadening (FWHM) lead to significant distortions in the overall results.

\subsection{Experiment}

The IRFs (in)directly determined from ${ }^{207} \mathrm{Bi}$ and ${ }^{60} \mathrm{Co}$ have been applied for the analysis of an experimentally obtained lifetime spectrum on $\alpha$-Fe5N. For subsequent comparison in terms of the retrieved parameter values and goodness-of-fit, the results obtained from the re-convolution fit using the analytical IRF modelled by 2 Gaussian functions prior obtained from the commonly applied NLSF technique, was taken as a reference. Even though the photon energy distributions (dominated by the Compton scattering) in the applied START and STOP PHS regions are equal for the three isotopes, deviations in the broadening (FWHM) remain existent causing distortions in the fit parameters, which reflect the tendencies as produced by the simulations. These deviations might originate from the different Compton edge energies of the isotopes leading to differently distributed photon (and electron) propagation angles after the Compton scattering affecting the average travel distance and time between incident and detection. Additional deviations in the broadening on the ${ }^{22} \mathrm{Na}$ site might originate from pile-up events, which could not be fully suppressed by software-based filtering, moreover, considering the significant higher activity of ${ }^{22} \mathrm{Na}(25 \times)$. Furthermore, slight 
deviations have also been observed in the shape of the different IRFs. This might result from an insufficient reproducibility of the isotope placement in between the detectors, which causes variations in the effective area of illumination of the PMT photocathode.

In a nutshell, this analysis technique could represent a potential alternative to the most commonly applied NLSF technique provided that an almost accurate determination (or modelling) of the true underlying IRF is assured. We have presented the limitations of this technique in relation to plastic scintillators, since an almost congruent energy distribution and, thus, a comparable setup resolution (FWHM) mainly dominated by the TTS of the PMTs can be configured by a correct choice of the PHS windows.

Further studies will include:

- different PMT geometries for additional hardwarebased pile-up suppression,

- comparable activities $(1-5 \mu \mathrm{Ci})$ for the applied isotopes: ${ }^{22} \mathrm{Na},{ }^{60} \mathrm{Co}$, and ${ }^{207} \mathrm{Bi}$,

- investigations on the sensitivity of the placement reproducibility of the isotopes in between the detectors regarding variations in the shape and FWHM of the resulting IRF.

A more detailed study will soon be published by the authors.

\section{Acknowledgments}

Financial support by the BMWiEn (Federal Ministry for EconomicAffairs and Energy) is acknowledged with the project StoryEV (03ETE011A). We thank Dr. M. Eldrup from Technical University of Denmark (DTU) for supporting us with rare literature.

\section{References}

[1] R.M. Nieminen, M.J. Manninen, in: Positrons in Imperfect Solids: Theory, Springer, Berlin 1979, p. 145 .

[2] A.A. Istratov, O.F. Vyvenko, Rev. Sci. Instrum. 70, 1233 (1999).
[3] R.A. Willoughby, SIAM Rev. 21, 266 (1979).

[4] P. Kirkegaard, M. Eldrup, Comput. Phys. Commun. 3, 240 (1972).

[5] P. Kirkegaard, M. Eldrup, Comput. Phys. Commun. 7, 401 (1974).

[6] J. Večeř, A.A. Kowalczyk, L. Davenport, R.E. Dale, Rev. Sci. Instrum. 64, 3413 (1993).

[7] D. Petschke, R. Helm, T.E.M. Staab, Data Br. 22, 16 (2019).

[8] D. Petschke, dpscience/DDRS4PALS: DDRS4PALS (Version 1.08), Zenodo, 2019.

[9] D. Petschke, T.E.M. Staab, SoftwareX. 10, 100261 (2019).

[10] D. Petschke, T.E.M. Staab, SoftwareX. 7, 122 (2018).

[11] D. Petschke, T.E.M. Staab, SoftwareX. 7, 171 (2018).

[12] D. Petschke, T.E.M. Staab, SoftwareX. 7, 259 (2018).

[13] D. Petschke, T.E.M. Staab, SoftwareX. 9, 183 (2019).

[14] F. Bečvář, J. Čížek, I. Procházka, Appl. Surf. Sci. 255, 111 (2008).

[15] F. Bečvář, J. Čížek, I. Procházka, J. Janotová, Nucl. Instrum. Methods Phys. Res. A 539, 372 (2005).

[16] F. Bečvář, Nucl. Instrum. Methods Phys. Res. B 261, 871 (2007)

[17] D. Petschke, T.E.M. Staab, Nucl. Instrum. Methods Phys. Res. A 162742 (2019).

[18] D. Petschke, dpscience/DLTReconvolution: DLTReconvolution v1.2 (Version 1.2), Zenodo, 2019.

[19] Y. Koechlin, A. Raviart, Nucl. Instrum. Methods 29, 45 (1964)

[20] S.O. Flyckt, C. Marmonier, Photomultiplier Tubes: Principles and Applications, Photonis, Brive (France) 2002, p. 265. 